\title{
Instrumentos para Avaliação Psicológica de Pessoas com Deficiência Visual: Tecnologias para Desenvolvimento e Adaptação
}

Instruments for the Psychological Assessment of Visually Impaired People: Technologies for Development and Adaptation

Instrumentos de Evaluación Psicológica de las Personas con Discapacidad Visual: Tecnologías para el Desarrollo y la Adaptación

Cassandra Melo Oliveira Universidade Federal de Santa Catarina

Carlos Henrique Sancineto da Silva Nunes Universidade Federal do

Rio Grande do Sul

http://dx.doi.org/10.1590/1982-3703001902013 
Resumo: A adaptação dos testes psicológicos para pessoas com deficiência é um processo de alta complexidade, o qual requer cuidados especiais na qualidade e acessibilidade dos instrumentos. Em se tratando da deficiência visual faz-se necessário atender a diferentes graus de comprometimento visual, os quais geralmente inviabilizam a realização dos testes psicológicos nos formatos tradicionais. Durante a adaptação dos instrumentos as tecnologias se tornam grandes aliadas, já que as Tecnologias Assistivas permitem que as pessoas com deficiência visual utilizem o computador e a Internet com praticamente as mesmas vantagens que os videntes. Deste modo, destaca-se a importância da modernização dos instrumentos psicológicos utilizando-se das tecnologias disponíveis no mercado. Contudo, quando estas são aplicadas nas adaptações dos testes apenas para grupos específicos, pessoas com certo tipo de deficiência e sem deficiência não se beneficiam dos instrumentos. Com o objetivo de minimizar tal limitação, foi proposto o Desenho Universal que busca a acessibilidade plena ou máxima possível. Assim, com a aplicação do Desenho Universal e das novas tecnologias no desenvolvimento de instrumentos psicológicos é possível contemplar uma população ampla, independentemente de suas variações corporais, potencializando a acessibilidade.

Palavras-chave: Adaptação. Instrumentos psicológicos. Deficiência visual. Tecnologias. Desenho Universal.

Abstract: The adaptation of psychological tests for people with disabilities is a highly-complex process, once it requires special attention with regard to instrument's quality and accessibility issues. When it comes to visual impairment, it is also necessary to consider its various degrees, whose characteristic, however, make impossible the development of psychological instruments in traditional formats. During the adaptation of psychological instruments, technologies become great allies, once assistive technologies allow people with visual disabilities to use computer and internet with the same extent of those individuals without impairment. Additionally, it is worth highlighting the importance of modernization of psychological instruments that use such technologies. Nevertheless, when those assistive technologies are applied within the adaptation of instruments for specific groups, people with certain types of disabilities, and even those without disabilities, do not benefit from these instruments. In order to minimize such limitation, universal design has been proposed, which aims to obtain full or maximum possible accessibility. Therefore, through the application of universal design principles, along with new technologies to psychological instruments, a broad population might be covered by those instruments, regardless of physical impairments, furthermore, maximizing their accessibility.

Keywords: Adaptation. Psychological instruments. Visual impairment. Technologies. Universal design.

Resumen: La adaptación de los tests psicológicos para las personas con discapacidad es un proceso muy complejo, que requiere un cuidado especial con la calidad de los instrumentos y con su accesibilidad. En el caso de la discapacidad visual, es necesario cumplir con los diversos grados de deterioro visual, que a menudo hacen que sea imposible alcanzar los tests psicológicos en los formatos tradicionales. Durante el ajuste de los instrumentos las tecnologías de apoyo se convierten en grandes aliados, ya que permiten a las personas con discapacidad visual utilizar la computadora y el Internet con prácticamente las mismas ventajas que los videntes. Por lo tanto, destacamos la importancia de la modernización de los instrumentos psicológicos que utilizan las tecnologías disponibles en el mercado. Sin embargo, cuando se aplican en las adaptaciones de los tests sólo a grupos específicos, las personas con ciertos tipos de discapacidades y sin discapacidades no se benefician de los instrumentos. A fin de minimizar esta limitación, el Diseño Universal se ha propuesto buscar la accesibilidad completa o máxima posible. Así, con la aplicación del Diseño Universal y las nuevas tecnologías en el desarrollo de instrumentos psicológicos se puede atender una amplia población, independientemente de sus cambios corporales, aumentando la accesibilidad.

Palabras clave: Adaptación. Tests psicológicos. Tecnologías. Diseño Universal. 


\section{Introdução}

Os instrumentos utilizados por psicólogos no Brasil, sobretudo os testes, são comumente utilizados em formatos tradicionais, tais como: lápis e papel, pranchas, ilustrações em papel ou placas de papel cartão ou madeira - sendo a tecnologia envolvida reduzida ou ultrapassada. Tal aspecto dificulta a sua utilização por pessoas com deficiência, pois a acessibilidade de instrumentos nesses formatos é limitada. Assim, a elaboração de meios de acessibilidade, os quais ultrapassem as barreiras impostas por materiais pouco acessíveis, apresenta-se como um desafio para os pesquisadores que trabalham com o desenvolvimento e adaptação do instrumental do psicólogo e, para tanto, os recursos tecnológicos tornam-se grandes aliados.

Ao desenvolver ou adaptar testes para pessoas com deficiência, os pesquisadores e desenvolvedores de testes psicológicos devem buscar garantir a acessibilidade dos materiais utilizados para uma ampla gama de deficiências, as quais possuem especificidades. O teste psicológico no formato tradicional apresenta barreiras para execução, dependendo do tipo de deficiência e do grau de comprometimento. Assim, muitas vezes, faz-se imprescindível o uso de adaptações que permitam que pessoas com deficiência sejam capazes de acessar o conteúdo de um instrumento. Os textos internacionais de Case, Zucker e Jeffries, (2005), Goodman, Evans e Loftin, (2011), British Psychological Society, (2007), American Educational Research Association, American Psychological Association e National Council on Measurement in Education, (1999) apontam para a escassez de estudos na área.

Com vistas a proporcionar a acessibilidade aos instrumentos de avaliação, são realizadas adaptações em seus materiais, formas de aplicação, bem como a utilização de Tecnologia Assistiva e outros recursos. Os objetivos deste artigo são, reconhecendo a importância das adaptações, discutir a aplicação de recursos tecnológicos como forma de possibilitar acessibilidade e apresentar a proposta do Desenho Universal como potencializadora da acessibilidade na adaptação e construção de instrumentos psicológicos de avaliação, com destaque para os testes psicológicos.

\section{Terminologia referente às adaptações}

Existem várias denominações nos textos internacionais a respeito das mudanças que são feitas em testes psicológicos ou outros instrumentos avaliativos com o objetivo de torná-los capazes de testar pessoas com alguma deficiência. Traduções possíveis dos termos e seus respectivos originais na língua inglesa são: acomodações (accommodation), modificações (modification) e adaptações (adaptations).

Segundo Case, Zucker e Jeffries (2005), os termos acomodações e modificações possuem definições diferentes; as acomodações são definidas como mudanças na maneira em que o teste é administrado, as quais não afetam a validade dos resultados nem o construto que está sendo avaliado. Nesta concepção, tais acomodações apenas auxiliam para que pessoas com deficiência demonstrem conhecimentos e habilidades no mesmo nível do que aquelas sem deficiência. Tal ponto de vista também é compartilhado por Goodman, Evans e Loftin (2011) para os quais as acomodações feitas nos testes são mudanças para fornecer ao testando o acesso ao teste e seus materiais.

As modificações, entretanto, são compreendidas pelos mesmos autores (Case, Zucker, \& Jeffries, 2005; Goodman, Evans, \& Loftin, 2011) como as mudanças que alteram o construto que está sendo medido como quando, por exemplo, se omite alguns itens ou partes inteiras de um teste por acreditar-se que não serão capazes de medir a habilidade desejada em uma pessoa com um tipo específico de deficiência. Desse modo, consideram que as modificações, diferentemente das acomodações, produzem resultados que não são comparáveis diretamente com os resultados do teste original (Case, Zucker, \& Jeffries, 2005; Goodman, Evans, \& Loftin, 2011). 
O termo adaptação é utilizado para qualquer modificação ou acomodação realizada em um instrumento, inclusive nos procedimentos de padronização e normatização. $\mathrm{O}$ termo adaptação também é utilizado indistintamente para instrumentos que tiveram apenas um aspecto modificado ou que foram feitas várias modificações (American Educational Research Association, 1999; British Psychological Society, 2007).

Nos Standards for educational and psycological testing (American Educational Research Association et al., 1999) é destacada a importância das acomodações, modificações e adaptações para tratar de mudanças nos testes no que se refere à adequação ao público com deficiência. Nos Standards, o termo acomodação trata de qualquer ação no sentido de permitir que pessoas com deficiência participem do processo de testagem e o termo modificação alude a uma alteração específica no teste, por exemplo, uma modificação no processo de aplicação do teste - como o tempo de testagem. Contudo, é explicitado que não se pretende dar nenhuma conotação de que modificações implicam necessariamente em mudanças no construto.

No cerne do debate sobre terminologias mais adequadas estão situadas questões teóricas e metodológicas mais amplas: Será que as mudanças realizadas na padronização dos testes para que possam ser utilizados por pessoas com deficiência interferem na sua validade e fidedignidade? E a normatização desses instrumentos precisa ser alterada ou refeita?

No campo da discussão sobre a expressividade das adaptações e sua repercussão nos testes, o British Psychological Society (2007) defende que as mudanças na padronização dos testes afetam a sua validade e fidedignidade já que a padronização dos testes contribui para a sua eficácia e objetividade. Ressaltam ainda que qualquer alteração na padronização de um instrumento pode invalidar os seus resultados - ao menos quando é adotada a leitura da Teoria Clássica dos Testes. Uma possível solução para este impasse apontado pelas instituições britânicas, referidas no início deste parágrafo, seria a utilização de modelos baseados na Teoria de Resposta ao Item. O uso de estratégias efetivas de equalização, por exemplo, permite que pessoas que tenham respondido a conjuntos não idênticos de itens tenham seus resultados estimados de forma comparável (Embretson \& Reise, 2000).

Índices de fidedignidade, validade e no construto que o teste mede podem sofrer alterações significativas quando um teste é alterado - por exemplo, quando é feita a mudança da forma escrita comum para o Braille (American Educational Research Association et al., 1999). Seria considerado pouco convincente afirmar que o resultado de uma modificação em um teste específico possa ser concomitantemente estendido para todos os testes que forem transcritos para o Braille de igual modo, iindependentemente do construto medido e do formato do teste. Estudos científicos que repliquem as modificações com vários tipos de testes psicológicos poderão chegar a conclusões neste sentido.

A ausência de estudos que afirmem, de forma consistente, em qual grau os testes são afetados pelas mudanças necessárias ao seu uso por pessoas com deficiência, faz com que se tenha que optar pela utilização dos termos modificação, adaptação e acomodação, sem diferenciá-los conceitualmente. Contudo, primar-se-á pelo termo adaptação por ser mais genérico e de fácil compreensão na língua portuguesa, bem como por ser utilizado na literatura internacional tanto para expressar as acomodações quanto as modificações indistintamente (American Educational Research Association et al., 1999; British Psychological Society, 2007).

\section{Formas de adaptação}

As adaptações são inegavelmente importantes, uma vez que elas geralmente permitem que testes psicológicos sejam acessíveis para pessoas com deficiência, isto é, que possam beneficiar-se adequadamente do seu potencial instrumental. Assim, deve-se buscar meios para que os testes mantenham sua qualidade psicométrica e para que as 
inferências produzidas com base nos seus resultados mantenham-se válidas quando usados por pessoas com deficiência. Assim, tais adaptações precisam ser estudadas e aprimoradas.

Segundo os Standards (American Educational Research Association et al.,1999), existem diversas formas de adaptações, tais como: a) as alterações no meio de apresentação do teste, bem como no texto de suas instruções e itens, por exemplo: quando o texto é transcrito para o Braille, impresso em tamanho maior ou modificado para um formato de aplicação computadorizado; b) o uso de apenas parte de um teste, por exemplo, em um teste no qual parte dos itens são apresentados e respondidos de forma oral e parte na forma escrita (lápis e papel); e c) o formato das respostas também podem ser adaptados, por exemplo, respostas gravadas em caso de algum comprometimento motor que impeça o testando de realizá-las na forma escrita.

Diferentes recursos tecnológicos podem ser utilizados com vistas a tornar os instrumentos psicológicos acessíveis às pessoas com deficiências. Este artigo tratará de forma específica os recursos tecnológicos voltados para as pessoas com deficiência visual, porém não ficará restrito a este público ampliando o olhar para a perspectiva da acessibilidade plena defendida pelo Desenho Universal (DU). O conceito de Desenho Universal está relacionado com a busca pela acessibilidade plena ou máxima possível para pessoas com diferentes tipos de deficiência ou sem deficiência, idosos e crianças. Deste modo, o Desenho Universal visa à acessibilidade para a variação corporal humana e não um projeto específico para um tipo de deficiência. O conceito de DU será ainda discutido de forma mais aprofundada neste artigo.

\section{Deficiência Visual}

É considerada como deficiência visual a diminuição da resposta visual que, mesmo após tratamento clínico ou cirúrgico ou uso de lentes corretivas, perdura um comprometimento irreversível. A diferenciação entre deficientes visuais cegos e portadores de visão subnormal depende de duas escalas oftalmológicas: a acuidade visual e o campo visual. Na cegueira ocorre uma alteração grave ou total em funções elementares da visão tais como: cor, distância, forma e tamanho (Amorin, 2006; Conde, 2005; Sá, Campos, \& Silva, 2007).

Existem várias definições para a cegueira, pois esta reúne pessoas com vários graus de visão residual. A cegueira, quando ocorre desde o nascimento, é considerada congênita e posteriormente a este, cegueira adquirida. A cegueira total ou amaurose pressupõe a completa perda da visão, nestes casos nem a percepção luminosa existe. Assim, a cegueira é uma deficiência visual, mas existem os mais variados graus de deficiência visual e sua expressão também varia enormemente dependendo de fatores que vão além do simplesmente fisiológico, mas que envolvem aspectos emocionais e da aprendizagem (Conde, 2005; Amorim, 2006).

A deficiência visual não pode ser reduzida a um fenômeno fisiológico, já que há uma complexidade de fatores que a perpassam: sociais, afetivos, econômicos, culturais, políticos, artísticos, educacionais e tecnológicos. Pessoas que não dispõem da visão, mas que receberam cuidados, educação e oportunidades de participar da vida social e cultural possuem um desenvolvimento diferenciado e superior àqueles que não tiveram as mesmas oportunidades (Amorim, 2006; Kastrup, Sampaio, Almeida \& Carijó, 2009).

A maneira como o cego percebe a deficiência visual influenciará sua capacidade de desempenhar as atividades do dia a dia e esta percepção está relacionada à sua história de vida e aprendizagem. Modelos familiares super-protetores influenciam negativamente o desempenho social do indivíduo enquanto famílias que permitem e estimulam a independência do membro cego proporcionam seu desenvolvimento pessoal e social de forma mais efetiva (Amorim, 2006; Kastrup et al., 2009). 


\section{Tecnologia e deficiência visual}

Relativamente às pessoas com deficiência, a tecnologia é aplicada de diferentes maneiras e as pesquisas concentram-se em aspectos diferenciados que dependem dos objetivos e da perspectiva teórica que se parte. Quanto às pessoas cegas, são comuns pesquisas que buscam o voltar a enxergar utilizando-se de tecnologias (óculos especiais, implantes de eletrodos, retinas artificiais, olhos biônicos) ou, dentro do mesmo princípio de retorno da visão, mas por meio biológico (implante de proteínas sensíveis a luz e estudos com células-tronco). Essa perspectiva se centra no modelo biomédico, no qual a busca pela cura é o foco central (Kendrick, 2009). Outros estudos tentam a transposição do enxergar para o tato, considerando este como substituto da visão (Allman, 2009; Kendrick, 2009; Toniolli, \& Pagliuca, 2003).

Do ponto de vista da tecnologia empregada na busca por acessibilidade, encontra-se um amplo leque de alternativas que focam no dia a dia com a deficiência, entre elas destacam-se os programas de computador, soluções baseadas em recursos de voz, recursos de auxílio à mobilidade (pisos táteis) e navegação (navegadores que utilizam GPS), bem como teclados de computador adaptados ao Braille e até as já estabelecidas e amplamente utilizadas bengalas de locomoção. As tecnologias citadas neste parágrafo abrangem, em sua maioria, as Tecnologias Assistivas que consistem em um conjunto de instrumentos e técnicas desenvolvidos especificamente para pessoas com deficiências com o objetivo de auxiliá-las a desempenhar suas atividades diárias integrando-se na sociedade (Tabela 1) (Associação Brasileira de Normas Técnicas, 2004; Galvão Filho, 2009).

O destaque da Tecnologia Assistiva é, em grande parte, reflexo dos avanços tecnológicos gerais e das Tecnologias de Informação e Comunicação (TIC). Entretanto, a Tecnologia Assistiva é tão antiga quanto a fabricação dos primeiros instrumentos pelo homem. São considerados recursos de Tecnologia Assistiva tanto uma bengala quanto potentes aparelhos ópticos utilizados por pessoas com baixa visão. Soluções simples e artesanais são consideradas, pela sua utilidade, como Tecnologia Assistiva, sem necessariamente possuírem um grande rebuscamento tecnológico, um exemplo é o "Painel de preguinhos" (Tabela 1) (Associação Brasileira de Normas Técnicas, 2004; Galvão Filho, 2009).

A maioria das ferramentas tecnológicas descritas na Tabela 1 tem alguma relação com o computador, seja imprimindo o conteúdo deste em Braille ou sendo um sintetizador de voz que permite que uma voz eletrônica leia o conteúdo que está na tela do computador ou, ainda, digitalizando livros para que assim possam ser lidos por sintetizadores de voz ou transcritos para o Braille. Enfim, a computação trouxe uma ampla gama de possibilidades de uso para as pessoas com deficiência visual.

A utilização do computador pelos deficientes visuais possibilitou uma revolução na informação disponível para este grupo, sobretudo para os cegos, os quais possuíam acesso apenas a textos transcritos para o Braille ou dependiam de pessoas que os lessem. O computador e a Internet possibilitaram aos deficientes visuais cegos o acesso à informação em tempo real sem a necessidade de um mediador da informação humano; o mediador é a máquina e o operador cego busca as informações de acordo com seus interesses e necessidades (Sá, 2006; Duarte, 2010).

Soma-se às vantagens trazidas pelo uso do computador e da Internet, descritas no parágrafo anterior, a possibilidade de textos escritos pelos deficientes visuais serem lidos por uma ampla variedade de pessoas que não necessariamente possuam alguma deficiência, que sejam leitores do Braille ou que estejam vinculados a alguma instituição de apoio as pessoas com deficiência. Agregando-se as Tecnologias Assistivas ao computador e à Internet possibilita-se aos deficientes visuais disporem de quase todas as vantagens e possibilidades que tais tecnologias trazem para os videntes (Sá, 2006; Duarte, 2010). 
Tabela 1. Caracterização das tecnologias assistivas.

\begin{tabular}{|c|c|}
\hline Denominação & Descrição \\
\hline Braille Falado & $\begin{array}{l}\text { Os dados que entram em Braille são eletronicamente } \\
\text { guardados e permitem escrever, revisar e editar. }\end{array}$ \\
\hline Linha Braille & $\begin{array}{l}\text { As linhas Braille são dispositivos utilizados com leitores de } \\
\text { telas, que servem para os usuários cegos e com baixa-visão } \\
\text { acessarem de forma táctil a informação contida no monitor. }\end{array}$ \\
\hline $\begin{array}{l}\text { Programa TGD (Tactile } \\
\text { Graphics Designer) }\end{array}$ & $\begin{array}{l}\text { É um software pedagógico criado para a geração de figuras } \\
\text { e/ou gráficos em Braille. }\end{array}$ \\
\hline Software GRAPHIT & $\begin{array}{l}\text { Programa para produção de gráficos a partir de equações } \\
\text { matemáticas. }\end{array}$ \\
\hline Virtual Magnifying Glass & $\begin{array}{l}\text { É uma lupa virtual que permite a ampliação da tela do } \\
\text { computador. }\end{array}$ \\
\hline Open Book & $\begin{array}{l}\text { Permite que as pessoas com deficiência visual possam } \\
\text { acessar e editar materiais impressos mediante um processo } \\
\text { de escaneamento e digitalização. O software com voz } \\
\text { sintetizada faz a leitura de todos os textos }\end{array}$ \\
\hline Jaws & $\begin{array}{l}\text { Oferece tecnologia de voz sintetizada em ambiente Windows } \\
\text { para acessar softwares, aplicativos e recursos na Internet. }\end{array}$ \\
\hline Impressora de pontos Braille & Imprime em Braille. \\
\hline Plástico estofável por calor & $\begin{array}{l}\text { Um desenho é criado em tinta escura sobre o plástico (por } \\
\text { exemplo, utilizando-se uma impressora de jato de tinta) } \\
\text { que, ao ser submetido ao calor, estufa as regiões escritas. }\end{array}$ \\
\hline Impressoras de relevos & $\begin{array}{l}\text { Imprime mapas, plantas, símbolos matemáticos, tabelas, } \\
\text { gráficos, músicas. É utilizada no desenvolvimento de } \\
\text { noções 2D. }\end{array}$ \\
\hline Folha de plástico (German Paper) & Para desenho (bidimensional). Ao escrever se obtém um relevo. \\
\hline Painel de preguinhos & $\begin{array}{l}\text { Para desenho (bidimensional). Por meio dele se "desenha" } \\
\text { com elásticos em um painel com pregos (utilizado para o } \\
\text { ensino da geometria). }\end{array}$ \\
\hline Magic & $\begin{array}{l}\text { É um ampliador de tela (de } 2 \text { a } 16 x \text { ) para ambiente } \\
\text { Windows e todos os aplicativos compatíveis. O software } \\
\text { também faz a leitura da tela através de voz sintetizada. }\end{array}$ \\
\hline
\end{tabular}

Fontes': Amorim, Carvalho, \& Menezes, 2004; sites: Bengala branca (1011) e Tecnologias Assistivas (2011).

\section{Como os deficientes visuais utilizam o computador e a Internet?}

A partir do final dos anos 1970, nos Estados Unidos, cresceu o movimento de informatização do Sistema Braille tendo como consequência o desenvolvimento de diversos recursos de Tecnologia Assistiva, tais como as impressoras e os teclados Braille e os softwares de leitura e ampliação da tela. Atualmente, no Brasil, os teclados comuns ainda são os mais utilizados por pessoas com deficiência visual, por estarem facilmente disponíveis no mercado e o custo ser menor (Nogueira, Cheade, \& Granadeiro, 2004).

Na utilização do computador e da Internet por este público, com frequência o mouse perde sua utilidade, já que é um instrumento guiado primariamente pela visão do usuário, sendo necessária a sua substituição por teclas alternativas no teclado. Do mesmo modo, quando se trata da Internet para os cegos, é imprescindível que existam possibilidades 
de navegação utilizando o teclado, uma vez que estes utilizam a voz dos leitores para guiarem sua navegação ao invés da visão, por exemplo, a tecla utilizada para navegação através dos links da Internet é o tab.

A navegação, por meio de telas sensíveis ao toque (touchscreens), comuns nos smartphones e tablets, é viabilizada não mais pela tecla tab, mas por "gestos de acessibilidade" como o número de toques na tela e movimentos de arrastamento para os lados. O leitor de tela, da mesma forma que no computador, guia as escolhas lendo o conteúdo que está disposto na tela. Com vistas a assegurar a acessibilidade na navegação, alguns aspectos devem ser observados pelos desenvolvedores de sites como o fato de que as cores não são úteis para transmitir significados aos deficientes visuais, sobretudo aos cegos, e que gráficos complexos e imagens sem texto de descrição são inacessíveis para este grupo.

Pessoas com baixa visão utilizam programas que ampliam o tamanho das letras, modificam a fonte e o contraste. A ampliação necessária, bem como o contraste, variam enormemente de acordo com as preferências e o grau de comprometimento da visão do usuário. É possível, ainda, a utilização de leitores de tela de forma conjunta com os programas que realizam ampliações ou a opção por um destes recursos isoladamente (W3C, 2008; Sá et al., 2007).

Os programas leitores de tela consistem em um tipo de Tecnologia Assistiva proporcionada por sistemas computacionais, os quais são fundamentais para utilização do computador por deficientes visuais, pois é por meio deles que pessoas cegas guiam a sua utilização do computador bem como a navegação na Internet (a leitura destes programas é veiculada, por exemplo, em conteúdos de sites, textos e pdfs). Contudo, essas ferramentas possuem limitações como a incapacidade de saltar textos intermediários ou anúncios a fim de manter o foco apenas no conteúdo principal, além da impossibilidade de descrever gráficos e imagens. Uma solução viável para a problemática em questão é a criação de textos alternativos e a simplificação de gráficos. $\mathrm{Na}$ seleção de textos e anúncios, são necessários outros programas e o desenvolvimento de sites acessíveis (W3C, 2008; Sá et al., 2007).

O Dosvox, o NVDA, o Virtual Vision, o Voice Over e o Jaws são exemplos de programas capazes de transformar o texto escrito na tela do computador em áudio-texto. Os leitores possuem diversos recursos de mediação desta navegação, os quais são realizados digitando-se comandos no teclado. O Dosvox, o NVDA e o Virtual Vision são sintetizadores de voz de desenvolvimento brasileiro, permitindo, os dois primeiros, o download gratuito na Internet. Já o Voice Over é uma ferramenta de acesso universal disponível em alguns produtos como computadores e iPads fabricados pela empresa Apple. O Jaws é uma ferramenta paga, de desenvolvimento internacional, mas com versões em português (Souza \& Santarosa, 2003; Oliveira, 2013).

Estudo realizado por Oliveira (2013) constatou que, na execução de um teste de personalidade informatizado on-line por pessoas com deficiência visual, o leitor de tela em conjunto com o computador pessoal foram os recursos tecnológicos mais utilizados. Dentre os leitores de tela, o Jaws alcançou maior difusão confirmando a preferência dos deficientes visuais do Brasil por este software, como já afirmado em Souza e Santarosa (2003). Em se tratando da qualidade destes leitores, os autores Oliveira (2013) e Silveira, Bassani e Reidrich (2007) observaram que o Jaws, o Virtual Vision e o NVDA atendem aos requisitos mínimos de acessibilidade, sendo os dois primeiros superiores (em recursos e qualidade das vozes sintetizadas) ao NVDA.

Na tentativa de proporcionar a acessibilidade às pessoas com deficiência ao utilizarem a Internet, a W3C (2008), no documento Web Content Accessibility Guidelines, traça recomendações para tornar o conteúdo da Web mais acessível. Suas orientações visam a tornar o conteúdo acessível a pessoas com uma ampla gama de deficiências, entre elas a cegueira e a baixa visão. É ressaltado no texto que a adoção destas diretrizes beneficia não só os usuários com deficiência, mas os usuários em geral, fator este que aponta para 
a perspectiva do Desenho Universal que será discutido mais adiante neste artigo.

\section{Tecnologia e instrumentos psicológicos}

A Tecnologia obteve avanços sem precedentes e as formas de comunicação e busca de informação alcançaram uma velocidade, nas últimas décadas, jamais vista. Entretanto, os recursos tecnológicos utilizados pelos psicólogos, e o próprio instrumental utilizado quando realizam avaliações psicológicas não se aproveitaram plenamente de tal evolução.

Predominantemente, no Brasil, os testes são utilizados no formato lápis e papel, e suas figuras e formatação persistem com as mesmas características antiquadas dos testes que os antecederam. Não se considera com esta afirmação que não se deva primar pela validade e padronização e que, para tanto, frequentemente, se faz necessária a manutenção de certas características dos instrumentos. Contudo, alguns materiais precisam de modernização, haja vista que na era dos touchscreens a manutenção de figuras com representações de pessoas de outra época ou não representativos do contexto brasileiro e crivos em papel vegetal contribuem para trazer complexidade desnecessária aos materiais, além de torná-los desinteressantes e ultrapassados.

Pietro (2010) destaca a importância do teste informatizado para a avaliação psicológica, ressaltando que a APA, em 1986, lançou uma normativa sobre o assunto. No Brasil, não existe regulamentação específica estabelecida pelo CFP (Conselho Federal de Psicologia). Ainda segundo Pietro (2010), os testes informatizados são aqueles nos quais todas as etapas envolvidas no teste são realizadas através do computador: a apresentação das instruções, os exemplos dos itens, os itens, os dados do avaliando, o registro das respostas, a codificação numérica das respostas, o armazenamento dos dados, a pontuação da prova e a emissão de relatórios. Algumas vantagens da utilização do computador são: a padronização é conduzida por um processo automático sem intervenções externas, a economia de tempo e pessoal durante a aplicação, o fácil armazenamento e correção dos testes, o fornecimento de feedback para o testando sobre o tempo, a adequação dos exemplos de acordo com a compreensão e as necessidades do testando, a criação de formas paralelas para pessoas que são avaliadas com frequência nas quais as questões são apresentadas de forma aleatória e, por fim, a flexibilidade na elaboração de material para estimulação (itens mais atrativos).

Os testes devem possuir suas qualidades psicométricas comprovadas por meio de estudos de validade, fidedignidade, padronização e normatização, devem ainda possuir utilidade, serem capazes de auxiliar os psicólogos em suas atividades de avaliação. Além disso, devem ser atrativos para os pacientes ou avaliandos para que haja engajamento no processo e que os mesmos participem motivados na realização destes instrumentos (Prieto, 2010).

\section{Aplicação de tecnologias na adaptação de instrumentos psicológicos para serem utilizados por deficientes visuais}

Testes informatizados são uma tecnologia de destaque na avaliação psicológica tendo em vista a sua utilidade, praticidade e possibilidade de realizar adaptações com vistas a torna-los mais acessíveis ao público com deficiência. Como por exemplo: a) adaptações da forma de aplicação: trocar a aplicação tradicional por uma informatizada com o auxílio de Tecnologias Assistivas como os leitores de tela; b) na forma de resposta: utilização do teclado do computador, mouse, microfone ou tela sensível ao toque (touchscreens); e c) adaptação do material de testagem: possibilidade de aumentar as letras; modificar a fonte e o contraste. A utilização do computador transforma o teste em uma plataforma flexível a adaptações.

As Tecnologias Assistivas são imprescindíveis neste processo, mas e se os testes fossem 
desenvolvidos já com vistas a estas adaptações? Se fossem pensados desde o princípio para serem utilizados por uma população ampla que agregasse pessoas com e sem deficiência? Esta é a perspectiva do Desenho Universal, o qual difere da Tecnologia Assistiva, pois a segunda pensa em um projeto especializado para pessoas com deficiência sendo muito específicos para cada tipo de deficiência e, geralmente, não é utilizado por pessoas sem deficiência. Entretanto, uma não invalida a outra, ou seja, as Tecnologias Assistivas, quando utilizadas num projeto de acessibilidade plena (Desenho Universal), complementam e potencializam as suas qualidades.

A utilização de adaptações em testes é uma tarefa complexa que necessita de auxílio técnico. O British Psychological Society (2007) aconselham que deve ser sempre procurado auxílio na editora do teste ou de um psicólogo que tenha experiência e competência reconhecidas na avaliação de pessoas com deficiência antes de se efetuar qualquer alteração no teste. Goodman et al. (2011) complementam especificando que cada adaptação deve ser planejada com antecedência e em concordância com a deficiência do testando. Ressaltam ainda a importância da participação de um profissional de reabilitação e, quando possível, do próprio autor do teste. Ao final da avaliação, devem ser documentadas todas as alterações realizadas possibilitando a clareza e ética que deve existir em processos avaliativos.

\section{O Desenho Universal}

A variabilidade das habilidades humanas é imensa, mesmo dentro de um mesmo tipo de deficiência há diferentes graus de comprometimento e diferentes necessidades de adaptação de um instrumento. Uma adaptação que atende a um público pode não atender a outro, já que as deficiências não são homogêneas. Muitas vezes, faz-se necessária a utilização de mais de uma adaptação para possibilitar acessibilidade a um público ou indivíduo. Como atender às diferenças individuais e, ainda, avaliar com qualidade? O Desenho Universal vem ao auxílio dos desenvolvedores de testes como uma proposta de solução deste impasse.

O Universal Design, traduzido no Brasil como Desenho Universal, surgiu nos Estados Unidos, na década de 1990, no campo da arquitetura eáreas afins, como consequência de mudanças sociais ocorridas ao longo do século XX. As mudanças demográficas, na legislação, os movimentos por igualdade, o movimento pelo fim das barreiras (Barrier-free design), a evolução da Engenharia da Reabilitação e da Tecnologia Assistiva, mudanças econômicas e as mudanças no clima social proporcionaram como consequência inevitável o surgimento das ideias que fazem parte do escopo do conceito de Desenho Universal (Story, Mueller, \& Mace, 1998).

O termo Universal Design foi cunhado por Ron Mace para denominar as ideias que circundavam o movimento por mais acessibilidade frente à diversidade das habilidades humanas. Reconheceu-se ser preciso criar alternativas para atender às necessidades de muitas pessoas diferentes e não apenas de um extrato da população ou "pessoas padrão" para quem eram exclusivamente desenvolvidos os espaços e produtos. $\mathrm{Na}$ década de 1990, Mace organizou um grupo de arquitetos, engenheiros e designers com o objetivo de estabelecer os princípios de acessibilidade plena que norteiam o Desenho Universal (Ostroff, 2001; Hanna, 2005).

O Desenho Universal consiste em projetar materiais, edificações, ambientes acessíveis para a maioria da população, independentemente de serem pessoas com deficiências ou não. Procura excluir a necessidade de adaptação ou de um projeto especializado para pessoas com deficiências e, em seu lugar, busca atender à variação corporal humana, potencializando a máxima acessibilidade (Story et al., 1998; Governo do Estado de São Paulo, 2010).

Em decorrência da relevância social da proposta do Desenho Universal, tal movimento disseminou-se por diversos campos, sobretudo nos Estados Unidos, como as artes, a saúde e a educação. Do campo da educação 
e, sobretudo, a partir de sua aplicação na avaliação educacional, o Desenho Universal alcançou a avaliação psicológica (Story et al., 1998), como detalhado a seguir.

\section{Desenho Universal na Avaliação Psicológica}

O Desenho Universal pode ser aplicado tanto ao processo de adaptação e desenvolvimento de testes psicológicos, como no delineamento dos processos avaliativos em toda sua complexidade. Quando aplicado ao desenvolvimento e adaptação de testes psicológicos, permite que sejam criadas ferramentas flexíveis que consideram a miríade das habilidades humanas. O Desenho Universal é útil no planejamento de processos avaliativos considerando o público-alvo e na forma como é possível proporcionar a máxima acessibilidade deste público às estratégias e materiais utilizados. Em se tratando das pessoas com deficiência, o uso de adaptações dos materiais de avaliação é fundamental, tornando-se extremamente eficaz e até indispensável neste processo o uso de tecnologias, sobretudo o computador, o qual permite a criação de uma plataforma flexível capaz de agregar diferentes tipos de adaptações (Thompson, Johnstone, \& Thurlow, 2002).

A literatura internacional destaca estudos promissores na área da construção e adaptação de instrumentos ao Desenho Universal (Case, 2003; Ketterlin-Geller, 2005; Dolan, Hall, Banerjee, Chun, \& Strangman, 2005). No Brasil, estudo realizado por Oliveira (2013) aplicou os princípios do Desenho Universal a um teste informatizado de personalidade alcançando o atendimento da acessibilidade para pessoas com e sem deficiência visual.

\section{Conclusão}

As possibilidades de modernização dos instrumentos psicológicos diante da variedade de recursos tecnológicos disponíveis no mercado são imensas. A não utilização destes recursos em benefício da ciência psicológica e da prática profissional é um retrocesso. Chegamos a um avanço tal na área tecnológica que não há mais como retroceder e as perspectivas futuras apontam para a tecnologização de todas as áreas, inclusive da Psicologia.

A tecnologia contribui enormemente para tornar os instrumentos psicológicos acessíveis, e ferramentas informáticas simples (programas de acessibilidade, sintetizadores de voz, etc.) podem ser utilizadas para viabilizar o uso de técnicas de avaliação por pessoas com deficiências, entre elas as com deficiência visual. Contudo, não só as pessoas com deficiência se beneficiam da busca por altos padrões de design e informatização dos testes psicológicos. Pessoas com os mais diferentes níveis de habilidades e variações corporais beneficiam-se da modernização e informatização dos instrumentos psicológicos, bem como da utilização de ferramentas disponibilizadas na Internet.

O computador e a Internet são grandes aliados para os desenvolvedores de testes psicológicos que procuram aplicar os princípios do Desenho Universal na construção de instrumentos mais acessíveis, uma vez que permitem criar ambientes que proporcionam maiores possibilidades de variação, isto é, maior flexibilidade no uso, escolha de formatos, tamanhos de fontes, além do fato de que admitem o acoplamento de Tecnologias Assistivas que venham a complementar e potencializar o Desenho Universal. 


\section{Cassandra Melo Oliveira}

Mestre em Psicologia pela Universidade Federal de Santa Catarina, Florianópolis - SC. Brasil. E-mail: casymelo@gmail.com

\section{Carlos Henrique Sancineto da Silva Nunes}

Doutorado em Psicologia pela Universidade Federal do Rio Grande do Sul, Porto Alegre - RS. Brasil.

Docente da Universidade Federal de Santa Catarina, Florianópolis - SC. Brasil.

E-mail: carloshnunes@mac.com

\section{Endereço para envio de correspondência:}

Rua Jornalista Tito Carvalho. 155. Trindade. Bloco Veneza, Ap. 408. CEP: 88040-480. Florianópolis - SC. Brasil.

Recebido: 06/09/2013, Aprovado: 11/06/2015. 
Allman, C. B. (2009). Test access: making tests accessible for students with visual impairments. a guide for test publishers, test developers, and state assessment personnnel. american printing house for the blind. Louisville, KY: American Printing House for the Blind. Recuperado em julho de 2011, de http://www.aph.org/tests/access2/access2.pdf

American Educational Research Association, American Psychological Association, National Council on Measurement in Education (1999). Standards for educational and psychological testing. Washington, DC: Author.

Amorim, E. S. M. S., Carvalho, J. L., \& Menezes, L. K. B. (2004). Educação de cegos mediada pela tecnologia. Recuperado em junho de 2012, de http://www.smec.salvador.ba.gov.br/site/ documentos/espaco-virtual/espaco-autorias/ artigos/educacao $\% 20$ de $\% 20$ cegos $\% 20$ mediada\%20pelas\%20tecnologias.pdf

Amorim, M. L. C. (2006). Construçãoe adaptação de um teste de atenção para indivíduos com deficiência visual: estudo baseado no teste de atenção de Bams. Dissertação de Mestrado, Faculdade de Desporto da Universidade do Porto. Porto, Portugal.

Associação Brasileira de Normas Técnicas - ABNT (2004). NBR 9050/2004. Acessibilidade a edificações, mobiliário, espaços e equipamentos urbanos. Rio de Janeiro, RJ: autor.

Bengala Branca. (2001). [Produtos]. Recuperado de http://www.bengalabranca.com.br/2011/ index3.php?pagina=home

British Psychological Society, Psychological Testing Centre (2007). Visual impairment and psychometric testing: practical advice for test users managing the testing of people who have signt disabilities. Leicester: The British Psychological Society. Recuperado em março de 2011, de https://ptc.bps.org. uk/sites/ptc.bps.org.uk/files/Documents/ Guidelines \%20 and \%20Information/ Visual \%20Impairment $\% 20$ and $\% 20$ Psychological\%20Testing.pdf

Case, B. J. (2003). Universal design: policy report. Boulder, CO: Pearson Education. Recuperado em março de 2011, de http://www.pearsonassessments.com/ UniversalDesig
Case, B. J., Zucker, S., \& Jeffries, J. L. (2005). A primer on assessing the visually impaired: assessment report. Boulder, CO: Pearson Education. Recuperado em março de 2011, de http://www.pearsonassessments.com/ NR/.../O/Visuallylmpaired

Conde, A. J. M. (2005). Definindo a cegueira e a visão subnormal. Rio de Janeiro, RJ: Instituto Benjamin Constant. Recuperado em julho de 2011, de http://www.ibc.gov. br/?itemid=94\#more

Dolan, R. P., Hall, T. E., Banerjee, M., Chun, E., \& Strangman, N. (2005). Applying principles of universal design to test delivery: the effect of computer-based read-aloud on test performance of high school students with learning disabilities. Journal of Technology, Learning, and Assessment, 3(7). Recuperado em junho de 2011, de http://ejournals.bc.edu/ojs/index. php/jtla/article/view/1660/1496

Duarte, A. G. P. (2010). Estudo sobre a escrita dos cegos nas listas de discussão do DOSVOX. Monografia, Universidade Federal do Rio de Janeiro. Rio de Janeiro, RJ. Recuperado em julho de 2011, de http://intervox.nce.ufrj.br/ dosvox/textos.htm

Embretson, S., \& Reise, S. P. (2000). Item response theory for psychologists (Multivariate applications book series, Vol. 4). New Jersey, NJ: Lawrence Erlbaum Associates.

Galvão Filho, T. A. A. (2009). Tecnologia assistiva: de que se trata? In G. J. C. Machado, \& M. N. Sobral (Orgs.). Conexões: educação, comunicação, inclusão e interculturalidade (pp. 207-235). Porto Alegre: Redes.

Goodman, S. A, Evans, G. C., \& Loftin, M. (2011). Position paper: intelligence testing of individuals who are blind or visually impaired. Louisville, KY: American Printing House for the Blind. Recuperado em março de 2011, de http://www.aph.org/tests/ intelligencetesting.html

Governo do Estado de São Paulo, Secretaria de Estado da Habitação, \& Secretaria de Estado dos Direitos da Pessoa com Deficiência (2010). Desenho universal: habitação de interesse social. Recuperado em março de 2011, de http://www.habitacao.sp.gov.br/ download/manuais-e-cadernos/manualdesenho-universal.pdf 
Hanna, E. L. (2005). Inclusive design for maximum accessibility: a practicalapproach to universal design. Boulder, CO: Pearson Educational Measurement. Recuperado em março de 2011, de http://www.pearsonassessments. com/NR/rdonlyres/BB1BC770-BCC1-4F069DA5-8D19A81E6C49/0/RR_05_04.pdf

Kastrup, V., Sampaio, E., Almeida, M. C., \& Carijó, F. H. (2009). O aprendizado da utilização da substituição sensorial visuo-tátil por pessoas com deficiência visual: primeiras experiências e estratégias metodológicas. Psicologia \& Sociedade, 21(2), 256-265.

Ketterlin-Geller, L. R. (2005). Knowing what all students know: Procedures for developing universal design for assessment. Journal of Technology, Learning and Assessment, 4(2). Recuperado em agosto de 2011, de http://t. www.edtechpolicy.org/ArchivedWebsites/ Articles/KnowingWhatAllStudents.pdf

Kendrick, M. (2009). Tasting the Light: device lets the blind "see" with their tongues. Scientific American. Recuperado em agosto de 2011, de http://www.scientificamerican.com/ article/device-lets-blind-see-with-tongues/

Nogueira, C., Cheade, C. F., \& Granadeiro, F. V. (2004). Sobre o braille. Rio de Janeiro: RJ; PUC-RIO. Recuperado em abril de 2013, de http://www.dad.puc-rio.br/dad07/arquivos_ downloads/31.pdf

Oliveira, C. M. (2013). Evidências de validade de uma bateria informatizada para avaliação da personalidade adaptada ao desenho universal. Dissertação de Mestrado, Universidade Federal de Santa Catarina, Florianópolis, SC.

Ostroff, E. (2001). Universal design: the new paradigm. In W. F. E. Preiser, \& E. Ostroff, Universal design handbook (1.3-1.11). New York, NY: McGraw-Hill Professional.

Pietro, G. (2010). Testes informatizados. In L. Pasquali. Instrumentação psicológica: fundamentos e práticas (pp. 467-489). Porto Alegre, RS: Artmed, 2010.

Sá, E. D., (2006). Oficina Educação Inclusiva no Brasil: diagnóstico atual e desafios para o futuro - Relatório sobre tecnologias assistivas e material pedagógico. Recuperado em abril de 2012, de www. bancodeescola.com

Sá, E. D., Campos, I. M., \& Silva, M. B. C. (2007). Atendimento educacional especializado: deficiência visual. Brasília, DF: MEC.

Silveira, C., Bassani, P. B. S., \& Reidrich, R. O. (2007). Avaliação das tecnologias de softwares existentes para a inclusão digital de deficientes visuais através da utilização de requisitos de qualidade. Novas Tecnologias na Educação., 5(1), 1-16.

Souza, A. P. \& Santarosa, L. M. C. (2003). Ambientes digitais virtuais: acessibilidade aos deficientes visuais. Novas Tecnologias e Educação, 1(1), 1-11.

Story, M. F., Mueller, J. L. \& Mace, R. L. (1998). The universal design file: designing for people of all ages and abilities. Raleigh,NC, North Carolina State University. Design. Recuperado em agosto de 2011, de http://www.eric. ed.gov/ERICWebPortal/search/detailmini. jsp?_nfpb=true\&_\&ERICExtSearch_ SearchValue_0=ED460554\&ERICExtSearch_ SearchType_0=no\&accno=ED460554

Tecnologia Assistiva. Produtos. Recuperado em julho de 2011, de http://www. tecnologia-assistiva.org.br/produtos. php?id_categoria $=57$.

Thompson, S. J., Johnstone, C. J., \& Thurlow, M. L. (2002). Universal design applied to large scale assessments (NCEO Synthesis Report, Vol. 44). Minneapolis, MN: University of Minnesota, National Center on Educational Outcomes. Recuperado em julho de 2012, de http://education.umn.edu/NCEO/ OnlinePubs/Synthesis44.html

Toniolli, A. C. S.; Pagliuca, L. M. F. (2003). Tecnologia tátil para a avaliação da dor em cegos. Revista Latino-Americana de Enfermagem, 11(2), 220-226.

W3C Recommendation. (2008). Web content accessibility guidelines (WCAG) 2.0. Recuperadoemjulhode2012, dehttp://www. w3.org/TR/2008/REC-WCAG20-20081211/ 